\title{
Immunomodulatory Effects of Taiwanese Neolitsea Species on Th1 and Th2 Functionality
}

\author{
Yin-Hua Cheng, ${ }^{1}$ Ying-Chi Lin, ${ }^{1,2,3}$ Ih-Sheng Chen, ${ }^{2}$ Sian-De Liu, ${ }^{2}$ Jih-Heng Li, $^{1,2}$ and \\ Chia-Chi Wang ${ }^{1,2,3}$
}

${ }^{1} \mathrm{PhD}$ Program in Toxicology, Kaohsiung Medical University, Kaohsiung 80708, Taiwan

${ }^{2}$ School of Pharmacy, College of Pharmacy, Kaohsiung Medical University, Kaohsiung 80708, Taiwan

${ }^{3}$ Department of Medical Research, Kaohsiung Medical University Hospital, Kaohsiung 80708, Taiwan

Correspondence should be addressed to Jih-Heng Li; jhlitox@kmu.edu.tw and Chia-Chi Wang; chiachiwang@kmu.edu.tw

Received 19 January 2017; Revised 8 May 2017; Accepted 24 May 2017; Published 11 July 2017

Academic Editor: Pushpa Hegde

Copyright ( 2017 Yin-Hua Cheng et al. This is an open access article distributed under the Creative Commons Attribution License, which permits unrestricted use, distribution, and reproduction in any medium, provided the original work is properly cited.

\begin{abstract}
Neolitsea species, medicinal plants belonging to Lauraceae, contain rich alkaloids, steroids, sesquiterpenoids, and triterpenoids which possess antimicrobial, antioxidant, and anti-inflammatory bioactivities. However, species differences in the immunomodulatory effects and evidence pertaining to the effects of Neolitsea species on adaptive immunity are scarce. This study aimed to evaluate the immunomodulatory properties of ten Taiwanese Neolitsea plants on T helper (Th) cell functionality, especially Th1 and Th2. Most of the 29 crude extracts of Neolitsea were not toxic to splenocytes, except $N$. buisanensis roots. N. aciculata and N. villosa leaf extracts possessed differential immunomodulatory effects on Th1/Th2 balance. N. aciculata var. variabillima and $N$. hiiranensis leaf extracts attenuated both Th1 and Th2 cytokines while $N$. konishii dramatically suppressed IFN- $\gamma$ production. As N. aciculata var. variabillima and N. konishii leaf extracts significantly attenuated Th1 functionality, we further evaluated their effects on CD4 cells under CD3/CD28 stimulation. $N$. aciculata var. variabillima significantly suppressed IFN- $\gamma$, IL-10, and IL-17, demonstrating the broad suppressive effects on T helper cells; $N$. konishii significantly suppressed IFN- $\gamma$ and IL-10 production, while the production of IL-17 was not altered. Collectively, these data demonstrated that leaf extracts of Taiwanese Neolitsea species contain phytochemicals with potentials to be developed as selective immunomodulators.
\end{abstract}

\section{Introduction}

T cells play a pivotal role in the immune responses. They participate in a wide range of immune responses through a complicated cytokine network and via cell-cell interaction with other cells. Interleukin-2 (IL-2), a major autocrine and/or paracrine $\mathrm{T}$-cell growth factor, is primarily produced by $\mathrm{T}$ helper cells and participates in the development and activation of T cells $[1,2]$. T helper type 1 (Th1) cells produce interferon gamma (IFN- $\gamma$ ) to regulate immune responses and inflammation against viral and intracellular bacterial infections and inhibit tumor formation via the stimulation of antibody production and the activation of macrophage, cytotoxic $\mathrm{T}$ lymphocytes (CTL), and natural killer cells [3, 4]. On the other hand, Th2 cells induce interleukin-4 (IL-4) production to mediate the activation and maintenance of the humoral and/ or allergy immune response against extracellular parasites, bacteria, allergens, and toxins [5]. The dysfunction of T cells, such as the imbalance of Th1/Th2 responses and abnormal immunostimulation, may lead to a variety of immune diseases. The excessive amounts of IFN- $\gamma$ have been associated with several Th1-mediated immune disorders, such as delayed type hypersensitivity [6], Crohn's disease [7], and multiple sclerosis [8]. Immunosuppressants, developed for the treatment of these overreactive immune responses, also decrease normal immune responses and thereby increase the susceptibility of the patients to infections $[9,10]$. Therefore, it is important to develop immunoregulators with less severe side effects. Natural compounds are under intensive investigation and showed promising progressions [11-13]. Several medicinal plants have been reported to regulate inflammatory responses in a variety of different animal models by 
TABLE 1: The possible bioactivities of Taiwanese Neolitsea plants used in this study.

\begin{tabular}{llc}
\hline Scientific name & Parts of plant used & Known possible bioactivities \\
\hline N. aciculata & Leaf, stem, and root & Antibacterial, antioxidant, and anti-inflammatory activities [38] \\
$\begin{array}{l}\text { N. aciculata var. variabillima } \\
\text { N. acuminatissima* }\end{array}$ & Leaf, stem, and root & Need to be studied \\
N. buisanensis & Leaf, stem, and root & Cytotoxicity [43] \\
N. daibuensis* & Leaf, stem, and root & Need to be studied \\
N. hiiranensis* & Leaf, stem, and root & Anti-inflammation [40] \\
N. konishii & Leaf, stem, and root & Anti-inflammatory, antimicrobial [34], and immunomodulatory activities [27] \\
N. parvigemma* & Leaf, stem, and root & Vasoconstriction [25], cardiotonic, and anti-inflammatory effects [41, 60] \\
N. sericea var. aurata & Leaf, stem, and root & Anti-inflammatory [39], antifungal [61], and antiplatelet activity [26] \\
N. villosa & Leaf and stem & Antiradical activity [62] \\
\hline
\end{tabular}

*Taiwanese endemic Neolitsea species.

attenuation of interleukin-2 (IL-2) and interferon- $\gamma$ (IFN- $\gamma$ ) production [14-18].

Neolitsea, small evergreen trees or evergreen shrubs in the family Lauraceae, consists of about 100 species distributed in the tropics, especially in Brazil and Southern Eastern Asia. In Taiwan, there are 12 Neolitsea species and four of them, including $N$. acuminatissima, $N$. daibuensis, $N$. hiiranensis, and $N$. parvigemma, are endemic [19]. Recent studies on the diversity of phytochemical structures and bioactivities revealed the application potential of Neolitsea plants in industrial and medical fields $[20,21]$. Parts of Neolitsea plants have been used in folk medicine for long periods of time in Asia. For example, the roots of $N$. aurata and the seeds of $N$. chuii are used to alleviate edema. The leaves of $N$. cambodiana are applied to treat furuncle and carbuncle. The roots of $N$. zeylanica have been shown to relieve rheumatic arthralgia [22]. Studies on the chemistry and pharmacology of Neolitsea species have led to the isolation and identification of more than 150 compounds including alkaloids, terpenoids, sterols, steroids and their derivatives, flavonoids, essential oils, and fatty acids with diverse activities $[21,23]$. The essential oils of $N$. pallens exhibited antioxidant and antibacterial activities [24]. Alkaloids of $N$. konishii possessed vasoconstricting effects on rat aorta [25]. Sesquiterpenes of the N. parvigemma showed inhibitory effects on platelet aggregation [26]. Our previous study has shown that leaf extracts of $N$. hiiranensis and its derived terpenoids possessed immunomodulatory effects via regulation of IFN- $\gamma$ production [27]. However, due to the complexity of compositions within Neolitsea species, it is still unclear how other Taiwanese Neolitsea species modulate the functionality of immune cells. The objective of this study aimed to examine the immunomodulatory effects of Taiwanese Neolitsea species on T-cell immunity.

To evaluate the immunomodulatory effects of Taiwanese Neolitsea species on T-cell immunity, we cultured and stimulated the mouse primary splenocytes with concanavalin A (ConA), a well-known T-cell mitogen, to stimulate cytokine production [28, 29]. Splenocytes are consist of antigenpresenting cells, B cells, and various type of T cells and have been widely used as primary immune cells for studying the functionality of T cells [30-33]. IL-2, IFN- $\gamma$, and IL-4 were evaluated to determine the effects of these plant extracts on Th1/Th2 functionality. In the present study, leaf extracts, including $N$. aciculata var. variabillima, N. acuminatissima, N. hiiranensis, N. konishii, and N. villosa, significantly inhibited IFN- $\gamma$ production. These Neolitsea extracts showed potentials to be developed as new therapeutic immunomodulators. The chemical components and mechanisms of these medicinal plants to modulate Th1 functionality warrants further investigation.

\section{Materials and Methods}

2.1. Reagents and Chemicals. All reagents were purchased from Sigma (St. Louis, MO) unless otherwise stated. Enzyme-linked immunosorbent assay (ELISA) sets for cytokine measurement were purchased from BD Biosciences (San Diego, CA). Fetal bovine serum (FBS) and cell culture supplies were from Hyclone (Logan, UT).

2.2. Plants and Extraction. Twenty-nine crude extracts were prepared and extracted with cold $\mathrm{MeOH}$ at room temperature [34]. These plants used here were identified by one of the authors, Prof. Ih-Sheng Chen, and the voucher specimens were deposited in the Herbarium of the College of Pharmacy, Kaohsiung Medical University, Kaohsiung, Taiwan. Traditional usage and known possible bioactivities of these 29 Neolitsea extracts were shown in Table 1.

2.3. Animals. Male BALB/c mice (five weeks old) were obtained from BioLasco (Ilan, Taiwan). On arrival, mice were randomly transferred to plastic cages containing aspen bedding and quarantined for at least 1 week. Mice were housed in a temperature $\left(22^{\circ} \mathrm{C} \pm 2^{\circ} \mathrm{C}\right)$-, humidity $(50 \% \pm 20 \%)$-, and light ( $12 \mathrm{~h} \mathrm{light/dark} \mathrm{cycle)-controlled} \mathrm{environment.} \mathrm{Food}$ and water were supplied ad libitum. All animal experimental procedures and housing have been approved by the Institutional Animal Care and Use Committee (IACUC) of Kaohsiung Medical University, Taiwan (Protocol ID: 106014). The experimental mice were euthanized by carbon dioxide mixed with oxygen for anesthesia with approved IACUC protocol and regulations. 
2.4. Splenocyte Isolation. The mice were sacrificed, and their spleens were harvested and made into single-cell suspensions. The isolation of splenocytes was according to the previous procedures which have been described in detail before $[35,36]$. Briefly, spleens were gently dissociated by teasing on a sterile 60-mesh steel screen (Sigma-Aldrich). The cell suspensions were washed in incomplete RPMI 1640 medium (Hyclone, Logan, UT) supplemented with 5\% fetal bovine serum (Gibco, USA) and 1\% penicillin and streptomycin (Amresco). Lymphocytes were enriched by removing red blood cells from splenocytes after treating with ACK lysis buffer. To activate splenocytes, cells were seeded in 48-well plates and stimulated with $5 \mu \mathrm{g} / \mathrm{mL}$ of ConA (Sigma-Aldrich, St. Louis, MO). The splenocytes $\left(5 \times 10^{6}\right.$ cells $\left./ \mathrm{mL}\right)$ were either left untreated (control group) or exposed to crude extracts of Neolitsea extracts followed by stimulation with ConA $(5 \mu \mathrm{g} / \mathrm{mL})$ for $48 \mathrm{~h}$. The supernatants of culture wells were collected to detect the cytokine levels by enzymelinked immunosorbent assay (ELISA).

2.5. Cell Viability. Splenocytes $\left(5 \times 10^{6}\right.$ cells $\left./ \mathrm{mL}\right)$ were seeded into 96-well plates. The cells were either left untreated or treated with crude extracts followed by stimulation with ConA for $48 \mathrm{~h}$. The viability was determined by the 3-(4,5-dimethylthiazol-2-yl)-2,5-diphenyl-tetrazolium bromide (MTT) assay. A methyl-thiazol-tetrazolium stock solution $(5 \mathrm{mg} / \mathrm{mL}$ in phosphate-buffered saline) was then added to each well $(10 \mu \mathrm{L} /$ well $)$ and incubated for $4 \mathrm{~h}$. The formed formazan was dissolved in a lysis buffer (10\% SDS in N,N-dimethylformamide) overnight in the dark. The optical density was measured at $570 \mathrm{~nm}$ (and at $630 \mathrm{~nm}$ as a background reference) using a microplate reader (Dynatech Laboratories Inc., Chantilly, VA).

2.6. Isolation of CD4 T Helper Cells. Mouse CD4 T helper cells were purified from splenocytes by using magnetic cell separation with the CD4 T Lymphocyte Enrichment Kit (BD Biosciences). To induce the polarization of Th cells, CD4 T cells $\left(5 \times 10^{5}\right.$ cells $\left./ \mathrm{mL}\right)$ were seeded in the 48 -well flat bottom tissue culture plates which were precoated with antimouse CD3 antibody $(1 \mu \mathrm{g} / \mathrm{mL})$ overnight, and the cells were either left untreated or treated with Neolitsea extracts followed by stimulation with soluble anti-mouse CD28 antibody $(1 \mu \mathrm{g} / \mathrm{mL})$ for $48 \mathrm{~h}$. The culture supernatants and the cells were collected for ELISA assay and intracellular cytokine staining, respectively.

2.7. Measurement of Th1/Th2 Cytokines of Total T Cells and CD3/CD28-Stimulated CD4 T Cells by Enzyme-Linked Immunosorbent Assay (ELISA). To examine the immunomodulatory activities of $N$. species on the total T cells, the splenocytes $\left(5 \times 10^{6}\right.$ cells $\left./ \mathrm{mL}\right)$ and CD4 T cells were cultured in 48 -well plates $(300 \mu \mathrm{L} /$ well $)$ and either left untreated or treated with crude extracts followed by stimulation with ConA $(5 \mu \mathrm{g} / \mathrm{mL})$ or $\mathrm{CD} 3 / \mathrm{CD} 28$ stimulation for $48 \mathrm{~h}$. The supernatants were harvested and quantified for IL-2, IFN- $\gamma$, IL-4, IL-10, and IL-17 by sandwich ELISA kits according to the manufacturer's instructions (BD Biosciences).
2.8. Intracellular Cytokine Staining by Flow Cytometry Analysis. CD4 T cells were cultured in a 48-well plate and treated with $N$. species for $36 \mathrm{~h}$. For analysis of intracellular cytokine production, the cells then treated with GolgiStop $(0.6 \mathrm{~mL} / \mathrm{mL}$; BD Biosciences) for $10 \mathrm{~h}$ prior to being harvested for antibody staining. The CD4 T cells then were fixed and permeabilized using Fixation and Perm/Wash buffers (BD Biosciences) before intracellular IFN- $\gamma$ and IL- 4 staining by PE-conjugated anti-mouse IFN- $\gamma$ and IL- $4 \mathrm{mAb}$ (clone XMG1.2 and 11B11; Biolegend). Ten thousand of CD4 T cells were acquired on a BD LSR II flow cytometer (BD Biosciences). The mean fluorescence intensity (MFI) of IFN- $\gamma$ in total CD4 $\mathrm{T}$ cells was quantified by gating CD4 $\mathrm{T}$ cells and then analyzed using FlowJo software (Treestar, Inc., CA).

2.9. Statistical Analysis. All the data were analyzed using a GraphPad software Prism 5.0 (GraphPad Software Inc.) San Diego, CA, USA). Each treatment group was measured in quadruplicate and the data were presented as the mean \pm standard error (SE). Data were analyzed using a one-way analysis of variance (ANOVA) for multiple comparisons and Dunnett's two-tailed $t$-test was used to compare the results for the treatment groups with vehicle control group. $p<0.05$ was defined as statistically significant.

\section{Results}

3.1. The Effects of Crude Extracts of Neolitsea (N.) Species on Cell Viability and IL-2 Production by Murine Primary Splenocytes In Vitro. To evaluate the effects of $N$. species on splenocyte viability, we first investigated the direct cytotoxicity of $N$. species in vitro. Most of these crude extracts at the concentration of $10 \mu \mathrm{g} / \mathrm{mL}$ did not significantly affect the cell viability compared to vehicle control $(\mathrm{VH}$ was referred as $100 \%$ ), except for the roots of the N. buisanensis which reduced the cell viability by $17 \%$ (Figure 1 (a) and Supplemental Table 1 available online at https://doi.org/10.1155/ 2017/3529859). As IL-2 plays important roles in T-cell clonal expansion and activation, the supernatants of the treated groups were collected to determine the IL-2 levels. Most of the crude extracts of $N$. species did not alter IL-2 production, except for the roots of the $N$. hiiranensis and the leaves of the N. konishii which suppressed IL-2 production by $15 \%$ and $16 \%$ (at the concentration of $10 \mu \mathrm{g} / \mathrm{mL}$ ), respectively (Figure 1(b) and Supplemental Table 1). Collectively, these data showed that the root part of $N$. buisanensis is relatively toxic to primary immune cells compared to other extracts. It should be circumspectly considered for further development of the immunomodulatory ingredients from this plant. Moreover, the roots of the $N$. hiiranensis and the leaves of the N. konishii suppressed IL-2, revealing that these extracts may affect the maturation and early activation of $\mathrm{T}$ helper cells.

3.2. Neolitsea Crude Extracts Differentially Modulated Th1 and Th2 Cytokines Production. We next investigated the effects of crude extracts of $N$. species on ConA-stimulated cytokine production by splenocytes (Figure 2 and Supplemental Table 1). IFN- $\gamma$ and IL-4, which belong to the Th1 and Th2 signature cytokines, were determined to study the 


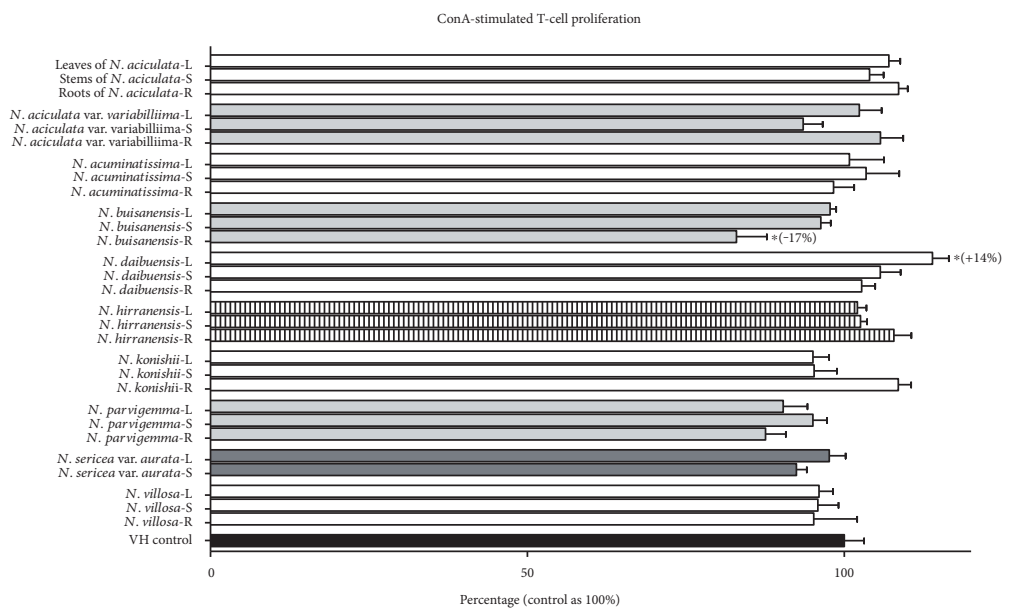

(a)

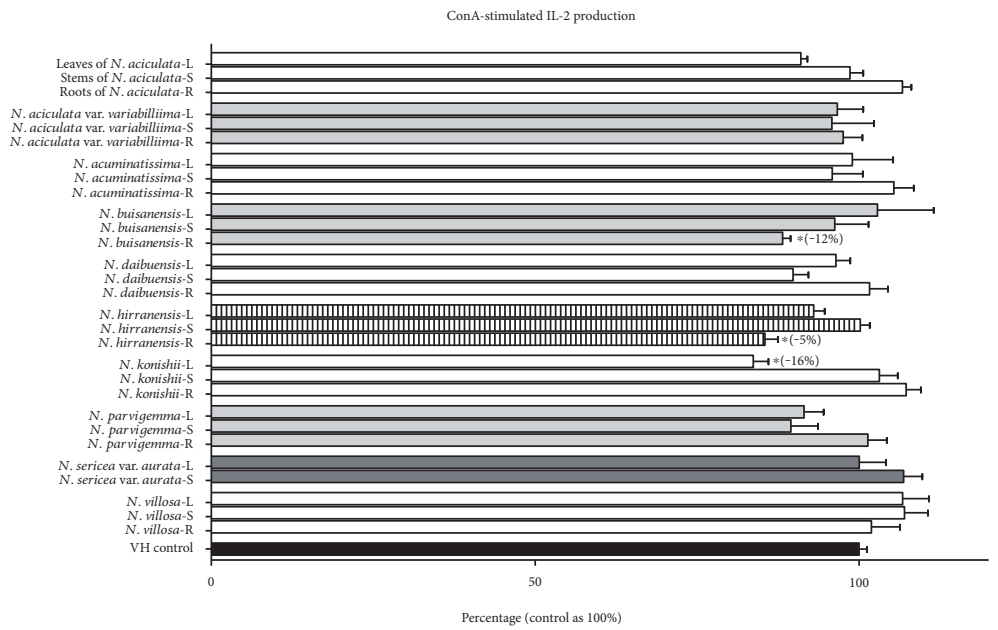

(b)

FIGURE 1: The effects of Taiwanese Neolitsea plants on T-cell viability and activation. Splenocytes $\left(5 \times 10^{6}\right)$ were treated with vehicle control $(\mathrm{VH}, 0.05 \% \mathrm{DMSO})$ and/or various crude extracts of Neolitsea plants $(10 \mu \mathrm{g} / \mathrm{mL})$ followed by ConA treatment for $48 \mathrm{~h}$. (a) The cell viability was determined using an MTT assay and the level of (b) IL-2 in the supernatants was quantified by ELISA. The viability and IL-2 level of the VH-treated group was shown as $100 \%$. L, S, and R were represented as leaf, stem, and root parts of different plants. The effects of N. aciculata, $N$. aciculata var. variabillima, $N$. acuminatissima, $N$. buisanensis, $N$. daibuensis, $N$. hiiranensis, $N$. konishii, N. parvigemma, N. sericea var. aurata, and N. villosa on (a) cell viability and (b) IL-2 production were shown from top to bottom, respectively. Data were expressed as the mean \pm SE of triplicated cultures. Results were pooled from three independent experiments. ${ }^{*} p<0.05$, compared to the VH group.

effects of plant-derived extracts $(10 \mu \mathrm{g} / \mathrm{mL})$ on $\mathrm{Th} 1 / \mathrm{Th} 2$ immune responses. N. aciculata did not affect the production of IFN- $\gamma$ and IL-4 except that the stem extracts of $N$. aciculata inhibited both IFN- $\gamma$ and IL- 4 by approximately $20 \%$ compared to $\mathrm{VH}$ control. These data suggested that the stem of $N$. aciculata inhibited both Th1 and Th2 functionality. The leaves and roots of $N$. aciculata var. variabillima (gray bar) significantly suppressed IFN- $\gamma$ production by $37-39 \%$, while the IL-4 was not altered. Interestingly, the stem of N. aciculata var. variabillima not only inhibited IFN- $\gamma(26 \%)$ but also inhibited IL-4 (17\%). These data indicated that Th1 cells were more sensitive to be suppressed by components of $N$. aciculata var. variabillima, and the leaf and root parts differentially modulated Th1 functionality. Different parts of $N$. acuminatissima (white bar) inhibited both IFN- $\gamma$ and IL-4 cytokine productions by $28-59 \%$ and $32-43 \%$, respectively. It was speculated that $N$. acuminatissima may affect the development of total Th cells in response to ConA stimulation. The leaves of $N$. buisanensis (gray bar) did not affect the IFN- $\gamma$ but reduced IL- 4 indicating the differential modulatory effects on Th2 functionality. N. hiiranensis attenuated IFN- $\gamma$ by $17 \%-90 \%$ and inhibited IL- 4 by $22-39 \%$. It is suggested that the whole extract of $N$. hiiranensis modulated both Th1 and Th2 activities (slash gray bar). The leaves and roots of $N$. konishii mainly attenuated IFN- $\gamma$ by $39-55 \%$ while IL- 4 was not affected. The leaf and stem parts of N. parvigemma inhibited Th1 and Th2 cytokine production by $22-$ $39 \%$ (gray bar). The leaves and stems of N. sericea var. aurata inhibited both IFN- $\gamma$ and IL-4 (22-28\% inhibition rate). Finally, N. villosa extracts attenuated IFN- $\gamma$ by $17-76 \%$, but IL-4 was not altered. Taken together, the above results showed that crude extracts of $N$. species differentially modulated $\mathrm{T}$ helper cell functionality. The leaf extracts of $N$. acuminatissima, N. hiiranensis, and N. parvigemma and $N$. 


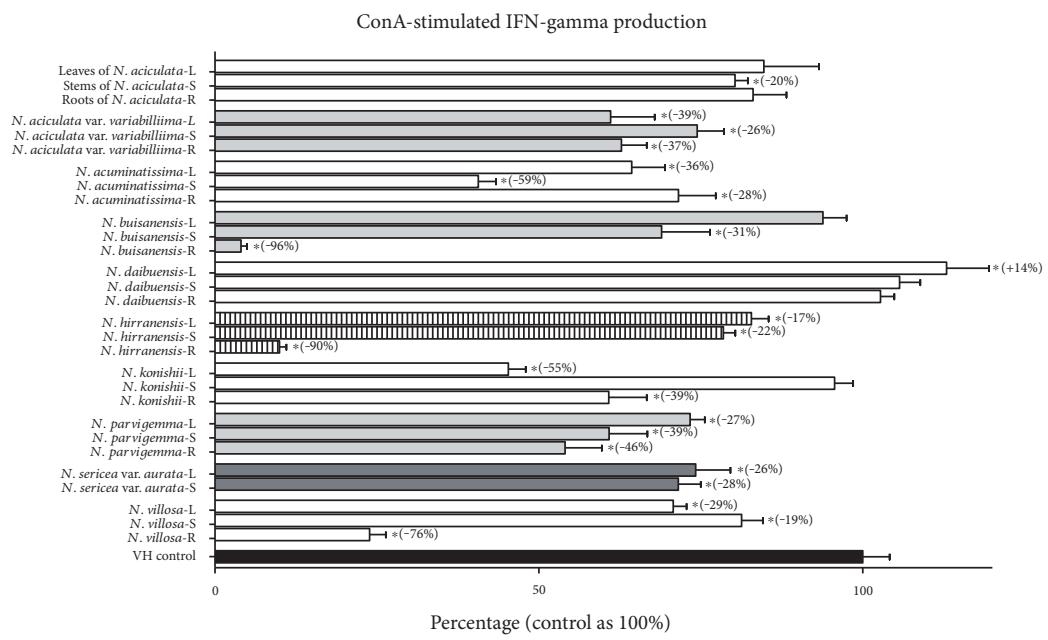

(a)

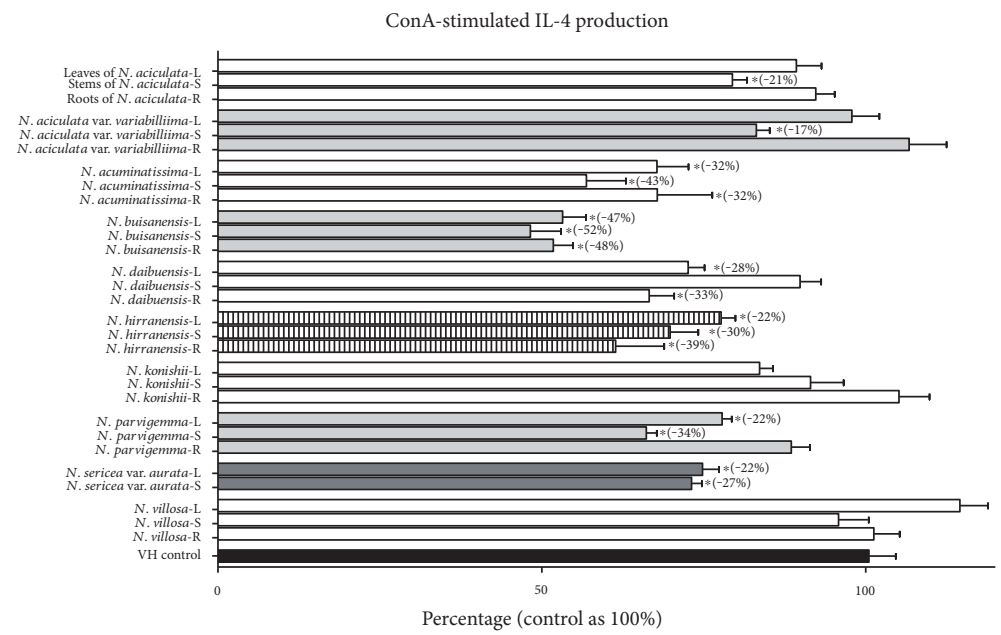

(b)

Figure 2: The effects of Taiwanese Neolitsea plants on Th1/Th2 cytokine production. Splenocytes $\left(5 \times 10^{6}\right)$ were treated with vehicle control $(\mathrm{VH}, 0.05 \% \mathrm{DMSO})$ and/or various crude extracts of Neolitsea plants $(10 \mu \mathrm{g} / \mathrm{mL})$ followed by ConA stimulation for $48 \mathrm{~h}$. The supernatants were collected for measuring the concentration of cytokines by ELISA. The cytokine level of the VH-treated group was shown as 100\%. L, S, and $\mathrm{R}$ were represented as leaf, stem, and root parts of different plants. The effects of $N$. aciculata, N. aciculata var. variabillima, N. acuminatissima, $N$. buisanensis, $N$. daibuensis, $N$. hiiranensis, $N$. konishii, $N$. parvigemma, $N$. sericea var. aurata, and $N$. villosa on (a) IFN$\gamma$ and (b) IL-4 were shown from top to bottom, respectively. Data were expressed as the mean \pm SE of triplicated cultures. Results were pooled from three independent experiments. ${ }^{*} p<0.05$, compared to the VH group.

sericea var. aurata suppressed both Th1 and Th2 functionality. N. aciculata var. variabillima, N. konishii, and N. villosa mainly inhibited IFN- $\gamma$ production. In contrast, $N$. buisanensis and $N$. daibuensis attenuated IL-4 production.

\subsection{The Differential Immunomodulatory Effects of Leaf and} Root Extracts of Selected Neolitsea Species on Th1/Th2 Functionality in Dose-Dependent Manners. According to our abovementioned results, several leaf extracts of Neolitsea species may differentially regulate the functionality of Th1 and Th2 cells by modulating the production of IFN- $\gamma$ and IL- 4 cytokines. We next further evaluated the concentrationdependent effects of these particular leaf extracts on ConAstimulated splenocytes. The effects of leaf extracts of $N$. aciculata, $N$. aciculata var. variabillima, $N$. daibuensis, $N$. hiiranensis, N. konishii, and N. villosa on the cell viability and
Th1/Th2 cytokine secretions were shown in Table 2. N. aciculata at the concentration of 5-50 $\mu \mathrm{g} / \mathrm{mL}$ did not alter the viability as well as IL-4 production. In contrast, $N$. aciculata concentration dependently inhibited IFN- $\gamma(25-50 \mu \mathrm{g} / \mathrm{mL})$ while IL-2 was suppressed at the concentration of $50 \mu \mathrm{g} / \mathrm{mL}$, indicating that leaf extracts of $N$. aciculata mainly affect Th1 functionality from $25 \mu \mathrm{g} / \mathrm{mL}$ (Table 2). N. aciculata var. variabillima $(5-50 \mu \mathrm{g} / \mathrm{mL})$ dramatically attenuated IFN- $\gamma$ production while IL-2 was suppressed at the concentration of $25-50 \mu \mathrm{g} / \mathrm{mL}$. N. aciculata var. variabillima $(50 \mu \mathrm{g} / \mathrm{mL})$ significantly inhibited cell viability and all tested cytokines demonstrating the cytotoxic effects of this plant at high concentration (Table 2). N. daibuensis (10-25 $\mathrm{g} / \mathrm{mL}$ ) attenuated IL-4 production while Th1 cytokines were not altered, demonstrating its differential effects on Th2 functionality (Table 2). N. hiiranensis did not affect cell viability and 
TABLE 2: The effects of $N$. species leaf extracts on Th1/Th2 cytokine production by ConA-stimulated splenocytes.

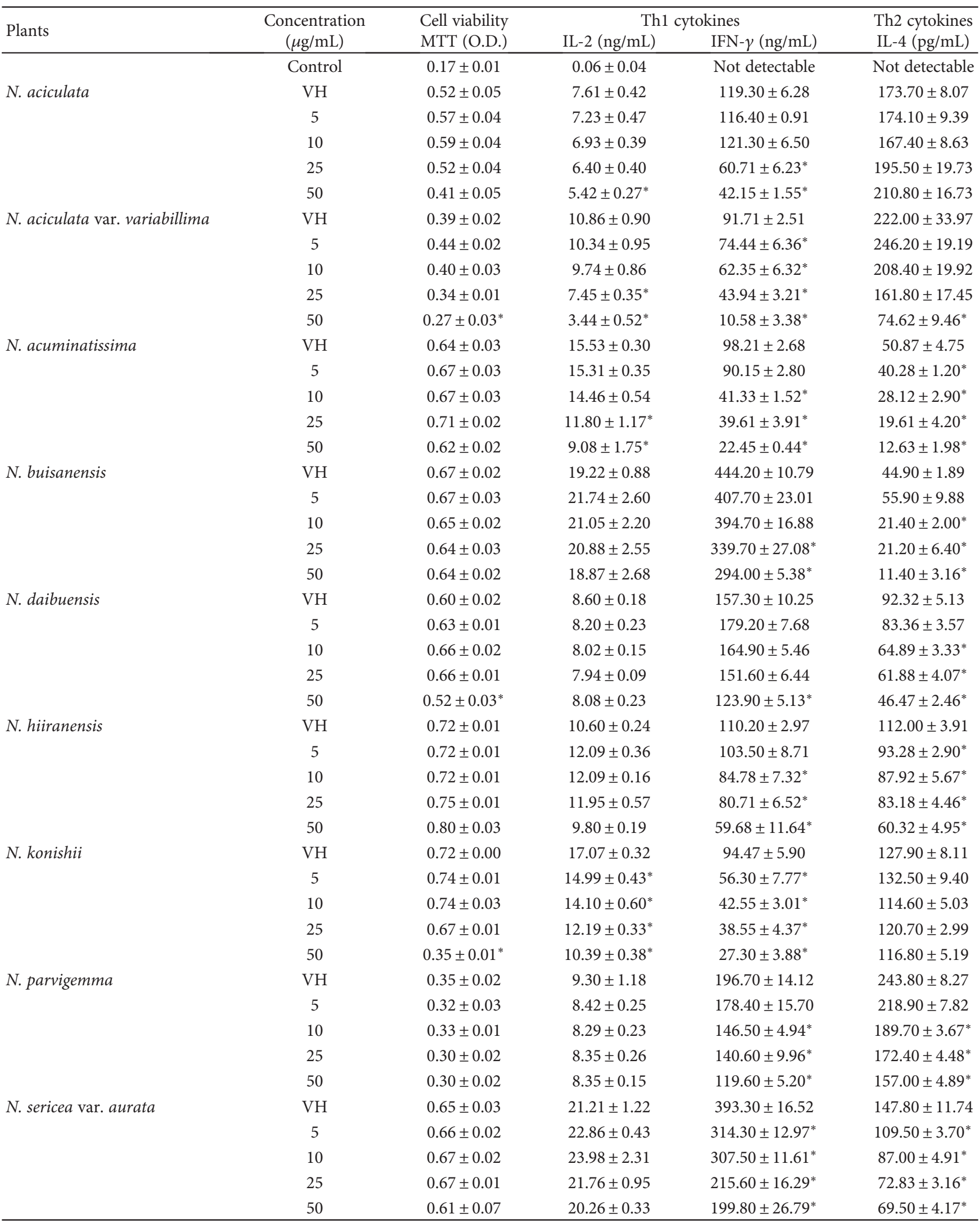


TABLE 2: Continued.

\begin{tabular}{|c|c|c|c|c|c|}
\hline \multirow{2}{*}{ Plants } & \multirow{2}{*}{$\begin{array}{c}\text { Concentration } \\
(\mu \mathrm{g} / \mathrm{mL})\end{array}$} & \multirow{2}{*}{$\begin{array}{l}\text { Cell viability } \\
\text { MTT (O.D.) }\end{array}$} & \multicolumn{2}{|c|}{ Th1 cytokines } & \multirow{2}{*}{$\begin{array}{l}\text { Th2 cytokines } \\
\text { IL-4 (pg/mL) }\end{array}$} \\
\hline & & & IL-2 (ng/mL) & IFN- $\gamma(\mathrm{ng} / \mathrm{mL})$ & \\
\hline \multirow[t]{5}{*}{ N. villosa } & VH & $0.47 \pm 0.03$ & $7.95 \pm 0.54$ & $87.60 \pm 4.38$ & $78.08 \pm 2.72$ \\
\hline & 5 & $0.42 \pm 0.01$ & $8.28 \pm 0.11$ & $77.24 \pm 7.08$ & $81.61 \pm 3.81$ \\
\hline & 10 & $0.44 \pm 0.01$ & $8.21 \pm 0.65$ & $61.96 \pm 1.89^{*}$ & $95.23 \pm 2.68$ \\
\hline & 25 & $0.52 \pm 0.02$ & $8.21 \pm 0.44$ & $50.67 \pm 8.33^{*}$ & $90.93 \pm 2.91$ \\
\hline & 50 & $0.55 \pm 0.02$ & $7.54 \pm 0.28$ & $29.95 \pm 4.15^{*}$ & $73.51 \pm 3.18$ \\
\hline
\end{tabular}

Data were expressed as the mean \pm SE of quadruplicate cultures. Results were representative of four independent experiments. ${ }^{*} p<0.05$ was significant compared to the VH group.

TABLE 3: The effects of $N$. species root extracts on Th1/Th2 cytokine production by ConA-stimulated splenocytes.

\begin{tabular}{|c|c|c|c|c|c|}
\hline \multirow{2}{*}{ Plants } & \multirow{2}{*}{$\begin{array}{c}\text { Concentration } \\
(\mu \mathrm{g} / \mathrm{mL})\end{array}$} & \multirow{2}{*}{$\begin{array}{l}\text { Cell viability } \\
\text { MTT (O.D.) }\end{array}$} & \multicolumn{2}{|c|}{ Th1 cytokines } & \multirow{2}{*}{$\begin{array}{l}\text { Th2 cytokines } \\
\text { IL-4 (pg/mL) }\end{array}$} \\
\hline & & & $\mathrm{IL}-2(\mathrm{ng} / \mathrm{mL})$ & IFN- $\gamma(\mathrm{ng} / \mathrm{mL})$ & \\
\hline \multirow{3}{*}{ N. aciculata var. variabillima } & Control & $0.06 \pm 0.00$ & $0.14 \pm 0.12$ & Not detectable & Not detectable \\
\hline & $\mathrm{VH}$ & $0.40 \pm 0.01$ & $10.15 \pm 0.71$ & $66.21 \pm 6.82$ & $166.50 \pm 5.30$ \\
\hline & 5 & $0.43 \pm 0.02$ & $9.31 \pm 0.31$ & $50.21 \pm 7.96$ & $171.00 \pm 18.73$ \\
\hline \multirow{8}{*}{ N. konishii } & 10 & $0.41 \pm 0.02$ & $10.32 \pm 0.22$ & $40.42 \pm 3.31^{*}$ & $210.20 \pm 18.04$ \\
\hline & 25 & $0.37 \pm 0.03$ & $8.02 \pm 0.11^{*}$ & $21.15 \pm 2.55^{*}$ & $141.20 \pm 7.61$ \\
\hline & 50 & $0.29 \pm 0.02^{*}$ & $5.90 \pm 0.31^{*}$ & $8.379 \pm 2.25^{*}$ & $86.02 \pm 6.78^{*}$ \\
\hline & $\mathrm{VH}$ & $0.68 \pm 0.00$ & $12.91 \pm 0.09$ & $99.63 \pm 8.09$ & $102.40 \pm 1.41$ \\
\hline & 5 & $0.69 \pm 0.01$ & $13.01 \pm 0.13$ & $64.55 \pm 4.33^{*}$ & $106.70 \pm 4.83$ \\
\hline & 10 & $0.72 \pm 0.01$ & $13.21 \pm 0.14$ & $48.06 \pm 6.32^{*}$ & $107.60 \pm 4.82$ \\
\hline & 25 & $0.71 \pm 0.03$ & $13.05 \pm 0.10$ & $25.46 \pm 2.70^{*}$ & $106.20 \pm 7.55$ \\
\hline & 50 & $0.64 \pm 0.01$ & $12.45 \pm 0.21$ & $7.93 \pm 1.34^{*}$ & $102.00 \pm 8.80$ \\
\hline
\end{tabular}

Data were expressed as the mean \pm SE of quadruplicate cultures. Results were representative of four independent experiments. ${ }^{*} p<0.05$ was significant compared to the $\mathrm{VH}$ group.

IL-2 production. Interestingly, both Th1 and Th2 cytokines were attenuated by $N$. hiiranensis leaf extracts (Table 2). $N$. konishii dramatically suppressed IFN- $\gamma$ at the concentration of $5-25 \mu \mathrm{g} / \mathrm{mL}$ while the cell viability was not altered. In addition, IL-2 production was suppressed from 5 to $25 \mu \mathrm{g} / \mathrm{mL}$. These data indicated the potential effects of $N$. konishii on Th1 functionality (Table 2). Interestingly, N. villosa at the concentration of $25 \mu \mathrm{g} / \mathrm{mL}$ slightly increased cell viability and IL-4 production while IFN- $\gamma$ was significantly suppressed. This data demonstrated the differential modulatory effects of $N$. villosa on Th1/Th2 balance (Table 2). As the leaf extracts of N. aciculata var. variabillima and N. konishii mainly inhibited IFN- $\gamma$ production, we further determined the effects of leaf extracts on IL-12 production and their root extracts on the functionality of T cells. The leaves of $N$. aciculata var. variabillima did not affect IL-12 production; however, N. konishii dramatically attenuated IL- 12 secretion at $25 \mu \mathrm{g} / \mathrm{mL}$, revealing that leaf extracts of $N$. konishii suppressed IFN- $\gamma$ production through the downregulation of upstream IL-12 production by splenic dendritic cells (Supplemental Fig.1). The root extracts of $N$. aciculata var. variabillima attenuated IL-2 and IFN- $\gamma$ production. The root extracts of $N$. konishii dramatically suppressed IFN- $\gamma$ at the concentration of $5-50 \mu \mathrm{g} / \mathrm{mL}$. These results demonstrated that both $N$. aciculata var. variabillima and N. konishii significantly suppressed Th1 functionality (Table 3).
3.4. The Direct Immunomodulatory Effects of Leaf Extracts of Selected Neolitsea Species on Th1/Th2 Functionality. Based on the above results, $N$. aciculata var. variabillima and $N$. konishii mainly modulated Th1 functionality in ConAstimulated splenocytes. We next analyzed the direct immunomodulatory effects of these two leaf extracts on CD4 T cells under stimulation of CD3 and CD28. CD3 is a major component of the T-cell receptor (TCR) complex, as well as CD28, which is a costimulatory molecule. The activation of both CD3 and CD28 will induce T-cell proliferation and cytokine production [37]. In addition, the production of IL-10 and IL-17 were determined to study the effects of these leaf extracts on the functionality of regulatory $\mathrm{T}$ cells and Th17 cells. The leaves extracts of $N$. aciculata var. variabillima significantly suppressed IFN- $\gamma$, IL-10, and IL-17 production at the concentration of $5-25 \mu \mathrm{g} / \mathrm{mL}$ in CD3/CD28 stimulated CD4 T cells (Figures 3(a), 3(c), and 3(d)), while IL-4 were slightly altered at the concentration of $25 \mu \mathrm{g} / \mathrm{mL}$ (Figure 3(b)). The leaf extracts of $N$. konishii mainly attenuated IFN- $\gamma$ and IL-10 productions at the concentration of 5$25 \mu \mathrm{g} / \mathrm{mL}$ (Figure 4), while IL-17 was not affected (Figure 4). We also performed the intracellular staining to detect the protein levels of IFN- $\gamma$ and IL-4 in CD4 T cells. Interesting, the leaf extracts of $N$. aciculata var. variabillima decreased the percentage of IFN- $\gamma^{+}$in CD4 T cells from $42 \%(\mathrm{VH})$ to $32 \%$, but the level of mean fluorescence intensity of 
$\mathrm{CD} 3 / \mathrm{CD} 28$-stimulated $\mathrm{CD} 4^{+}$cells

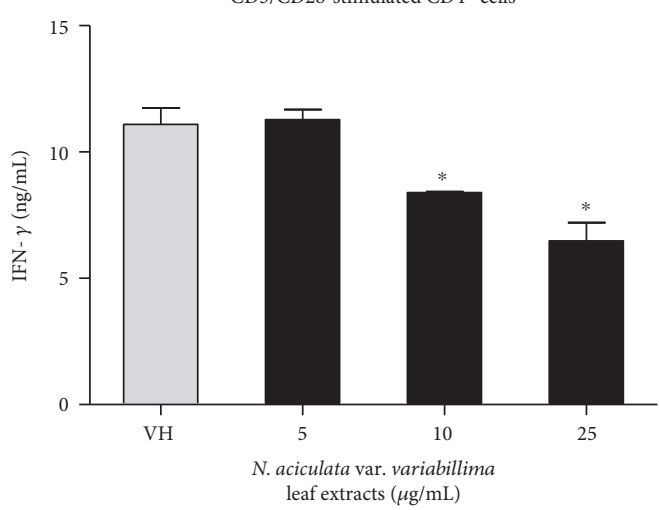

(a)

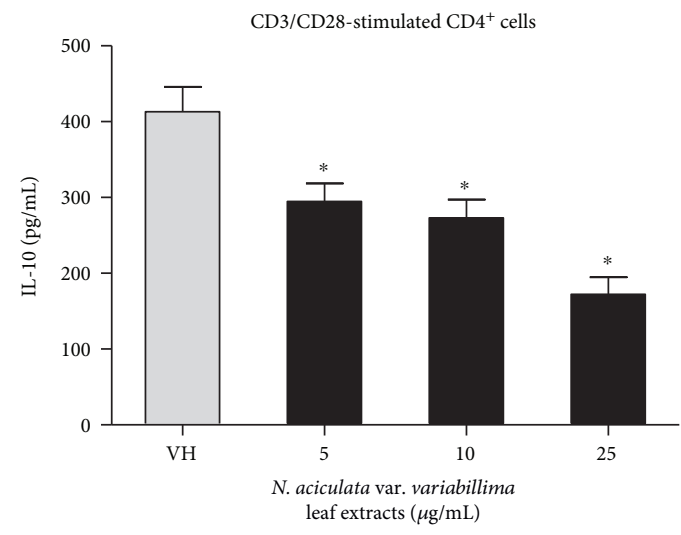

(c)

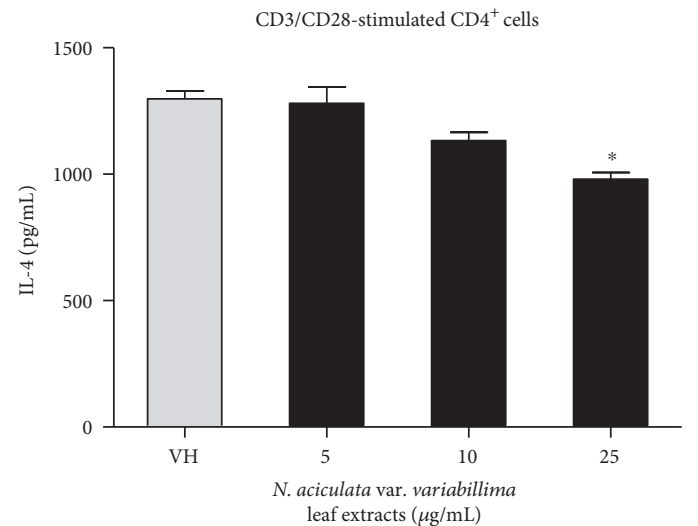

(b)

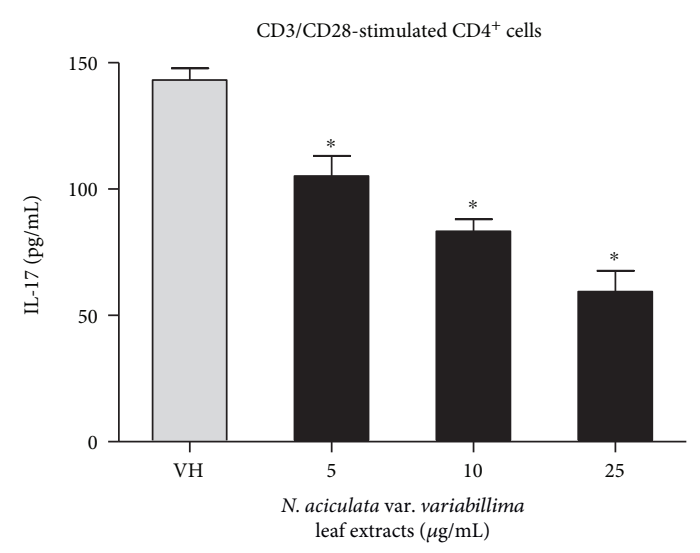

(d)
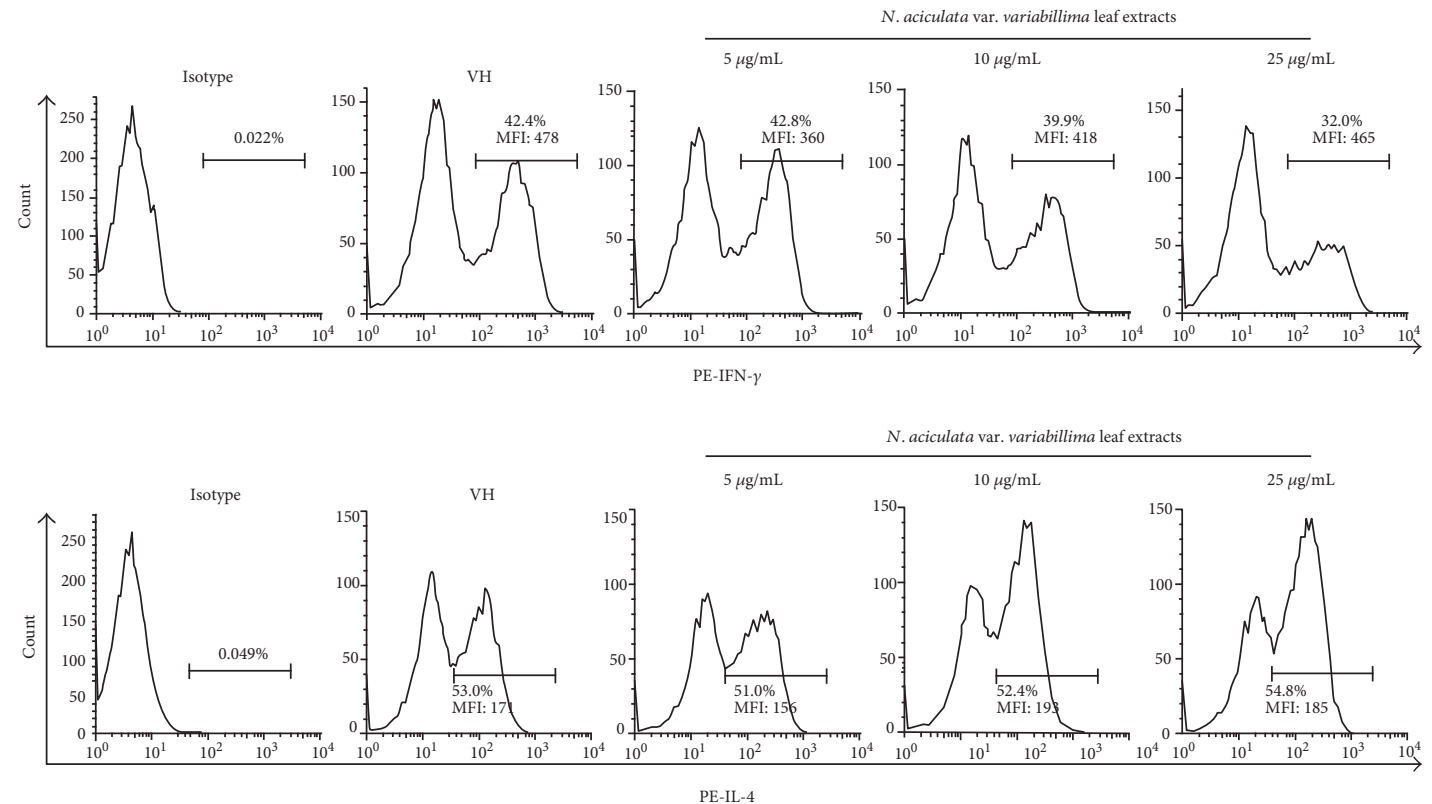

(e)

FIGURE 3: The effects of leaf extracts of $N$. aciculata var. variabillima on T-cell functionality. The enriched CD $4 \mathrm{~T}$ cells $\left(5 \times 10^{5}\right.$ cells $\left./ \mathrm{mL}\right)$ were stimulated with anti-CD3 and anti-CD28 $(1 \mu \mathrm{g} / \mathrm{mL})$ in the absence or in the presence of the leaf extracts of $N$. aciculata var. variabillima $(5-25 \mu \mathrm{g} / \mathrm{mL})$ for $48 \mathrm{~h}$. (a)-(d) The concentration of IFN- $\gamma$, IL-10, IL-4, and IL-17 in the supernatants was measured by ELISA. Data were expressed as the mean \pm SE of quadruplicate cultures. ${ }^{*} p<0.05$ was significant compared to the VH group. (e) The representative histogram of intracellular cytokine staining. Total percentage and the level of mean fluorescence intensity (MFI) of IFN- $\gamma^{+}$ and IL- $4^{+}$cells in $\mathrm{CD} 4^{+} \mathrm{T}$ cells were shown. Results were representative of two independent experiments. 


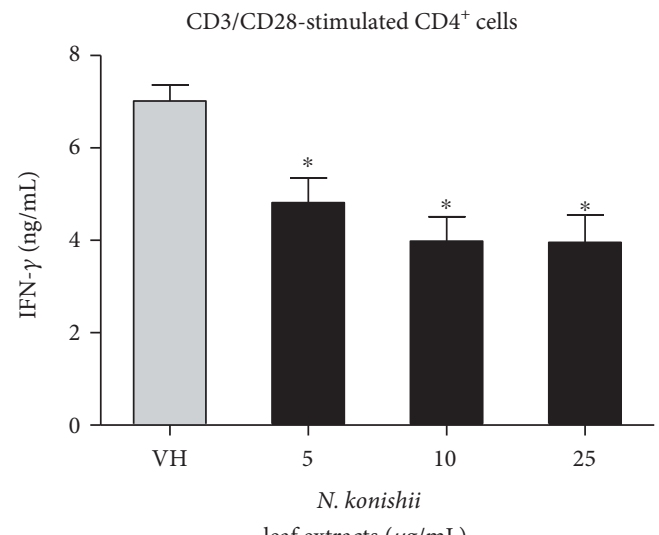

leaf extracts $(\mu \mathrm{g} / \mathrm{mL})$

(a)

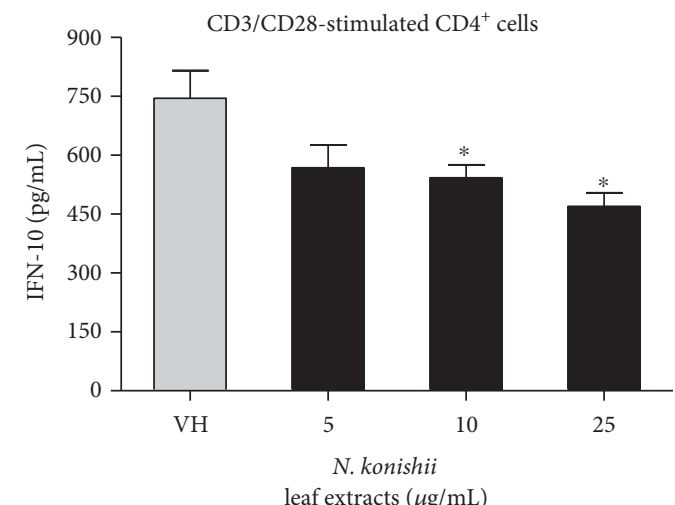

(c)
CD3/CD28-stimulated CD4 ${ }^{+}$cells

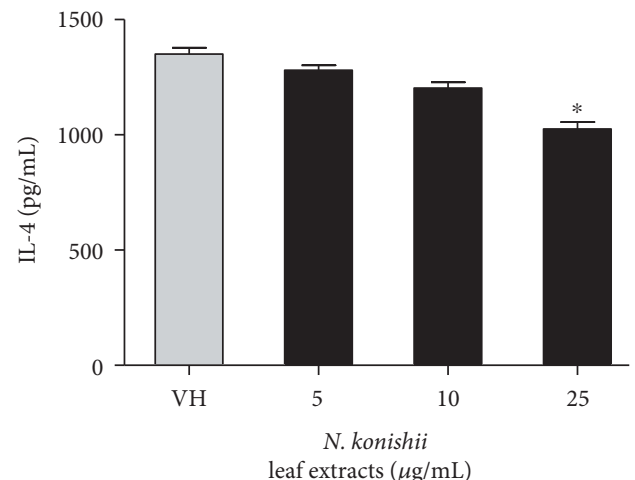

(b)

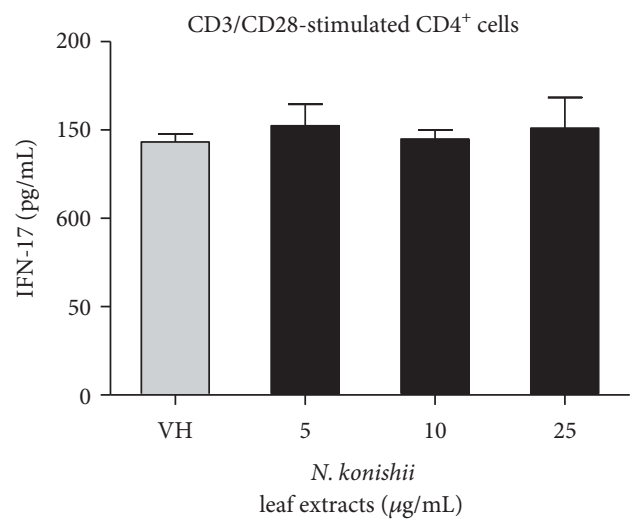

(d)
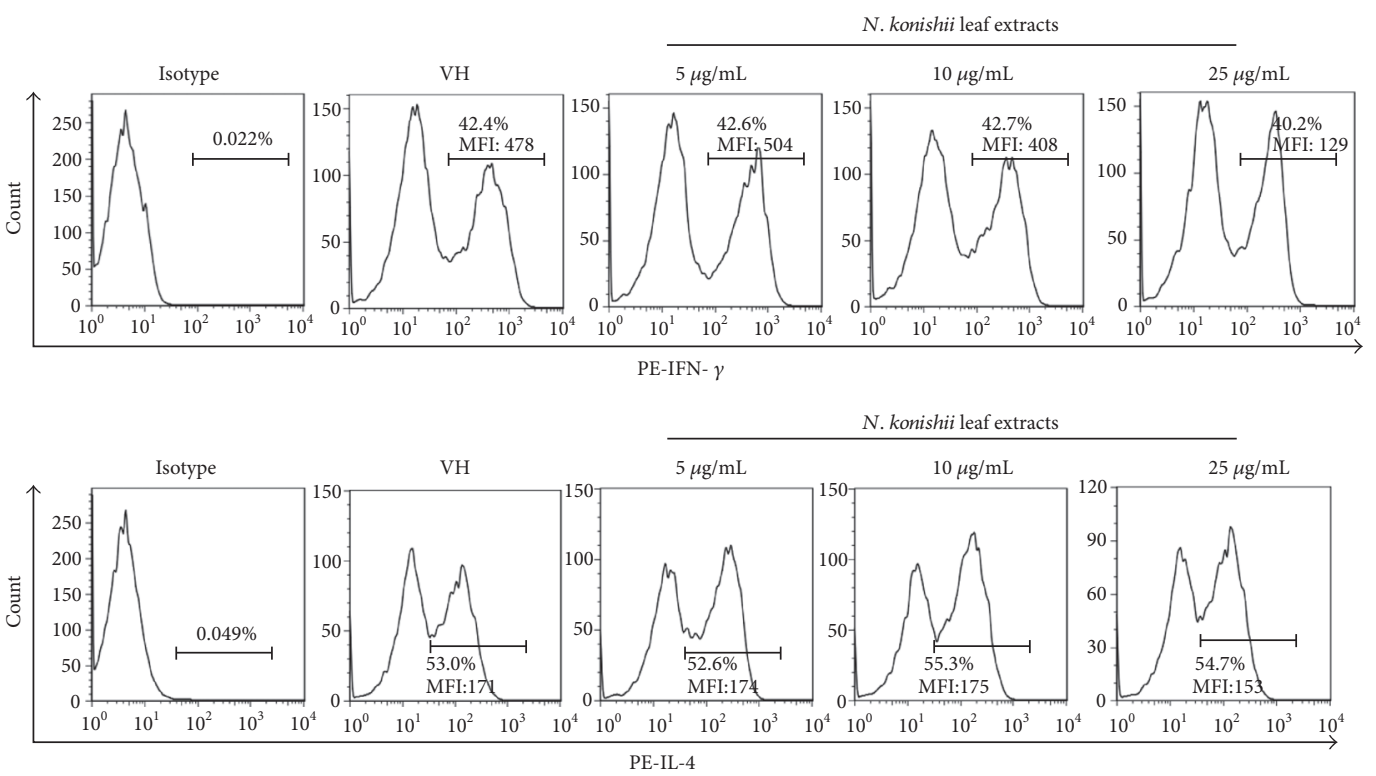

(e)

Figure 4: The effects of leaf extracts of $N$. konishii on T-cell functionality. The enriched CD4 T cells $\left(5 \times 10^{5}\right.$ cells $\left./ \mathrm{mL}\right)$ were stimulated with anti-CD3 and anti-CD28 $(1 \mu \mathrm{g} / \mathrm{mL})$ in the absence or the presence of the leaf extracts of $N$. konishii $(5-25 \mu \mathrm{g} / \mathrm{mL})$ for $48 \mathrm{~h}$. (a) $-(\mathrm{d})$ The concentration of IFN- $\gamma$, IL-10, IL-4, and IL-17 in the supernatants was measured by ELISA. Data were expressed as the mean \pm SE of quadruplicate cultures. ${ }^{*} p<0.05$ was significant compared to the $\mathrm{VH}$ group. (e) The representative histogram of intracellular cytokine staining. Total percentage and the level of mean fluorescence intensity (MFI) of IFN- $\gamma^{+}$and IL- $4^{+}$cells in CD $4^{+} \mathrm{T}$ cells were shown. Results were representative of two independent experiments. 
IFN- $\gamma$ was not significantly altered in IFN- $\gamma^{+}$cells. N. aciculata var. variabillima did not affect the percentage of IL- $4^{+}$ cells in CD4 T cells nor the protein levels of IL-4 in CD4 $\mathrm{T}$ cells, suggesting that the leaves of $N$. aciculata var. variabillima decreased the proportion of IFN- $\gamma^{+}$in CD4 T cells. By contrast, the leaf extracts of N. konishii attenuated the protein level of IFN- $\gamma$ in CD4 T cells while the proportion of IFN- $\gamma^{+}$cells were not changed.

\section{Discussion}

Natural products isolated from traditional medicinal plants have therapeutic effects in the prevention and treatment of various immune disorders. Neolitsea species exhibit extensive bioactivities and have been used as traditional herbal medicines in oriental countries. However, a comparative study of immunomodulatory properties of different Neolitsea species on immunocompetent cells such as $\mathrm{T}$ helper cells has not been demonstrated to date. Our previous reported study found that the leaf extracts of $N$. hiiranensis significantly inhibited IL-12, IFN- $\gamma$, and IL- 2 cytokine productions as well as the serum levels of OVA-primed antigen-specific IgM and $\operatorname{IgG}_{2 \mathrm{a}}$ in vivo [27]. In the present study, we evaluated the immunomodulatory properties of ten Taiwanese Neolitsea plants on T helper cells. Our results showed that most crude extracts of Taiwanese Neolitsea species decreased IFN- $\gamma$ production at concentrations below the $\mathrm{IC}_{50}$ by mitogenstimulated splenocytes, and the immunomodulatory activities of Neolitsea extracts, especially leaf extracts, were mainly on the suppression of Th1 immunity.

Several reports showed that Neolitsea species and its derived secondary compounds, including sesquiterpenoids, triterpenoids, alkaloids, and steroids, possess several bioactivities including anti-inflammatory activities $[18,20]$. Neolitsea aciculata essential oil (NAE) attenuated the Propionibacterium acnes-induced secretion of tumor necrosis factor-alpha (TNF-alpha) and interleukin-8 (IL-8) productions in human cell lines, revealing its anti-inflammatory effects [38]. Six furanogermacrane sesquiterpenes isolated from the stems of $N$. parvigemma, including deacetylzeylanidine, linderalactone, neolitrane, pseudoneolinderane, zeylanidine, and zeylanicine, have been shown to possess anti-inflammatory activities; among them, pseudoneolinderane and linderalactone have the ability to inhibit the superoxide anion generation by human neutrophils in response to formyl-l-methionyl-1leucyl-1-phenylalanine/cytochalasin B (fMLP/CB) with $\mathrm{IC}_{50}$ values of 3.21 and $8.48 \mu \mathrm{g} / \mathrm{mL}$, respectively [39]. Daibucarboline $\mathrm{A}$, isolinderalactone, 7-omethylnaringenin, and prunetin isolated from the roots of $N$. daibuensis exhibited moderate inhibition of inducible nitric oxide synthase (iNOS) [40]. Hiiranlactone $\mathrm{B}$ and hiiranlactone $\mathrm{D}$, isolated from the leaves of $N$. hiiranensis were classified as sesquiterpenes and possessed anti-inflammatory and immunomodulatory effects [27, 34]. Thaliporphine, the alkaloid of the bark extracts of N. konishii, demonstrated vasoconstricting effects by promoting $\mathrm{Ca}^{2+}$ entry [25] and possessed antioxidant, anti-inflammatory, and antiapoptotic activity to prevent cardiovascular system disorder in guinea pigs [41]. In addition to the antiinflammatory effects, the ingredients from Neolitsea species possess several bioactivities. Flavone glycosides, $2^{\prime}-p$-coumaroylafzelin and 2', $3^{\prime}$-di- $O$-( $p$-coumaroyl)afzelin, have been isolated from $N$. aciculata to demonstrate the antimelanogenesis activities [42]. Neolitacumone A-C and 2,6-dimethoxy- $p$ benzoquinone from the stem barks of $N$. acuminatissima displayed significant inhibitory activities against Hep 2.2.15 cells [43]. Isolinderalactone, a sesquiterpenes from the roots of N. villosa, exhibited antitumor activity [44]. Our recent study reported that $N$. hiiranensis-derived caryophyllene oxide inhibited several aspects of adaptive immune responses, including T-cell differentiation, IFN- $\gamma$ production, and Th1associated genes [27]. These studies suggested that the immunomodulatory effects of Neolitsea plants are worth further investigation; in addition, the present study aimed to clear how other Taiwanese Neolitsea species modulate the functionality of Th cells by ConA-stimulated splenocytes.

Th cells play pivotal roles in the acquired immunity. They are categorized into several subsets based on the cytokine production after stimulation [5]. The main roles of the type $1 \mathrm{Th}$ cells (Th1) are against intracellular bacteria and protozoa. They are predominantly induced by interleukin-12 (IL-12) and are fully activated by IL- 2 and IFN- $\gamma$. Induction of mitotic activity and cytokine production are associated with Th cell functionality. IFN- $\gamma$, an upstream cytokine of Th1 cells, regulates inflammatory immune response, promotes Th1 cell differentiation, enhances MHC class II expression on antigen-presenting cells, and possesses protective immune responses against cancer formation [4, 45-48]. Saha et al. reported that Viscum album-derived Qu Spez significantly stimulated IFN- $\gamma$ secretion showing its ability to modulate the immune system and to suppress tumor regression by regulation of dendritic cells [49]. Th1 cells secreted IFN- $\gamma$ to activate cell-mediated immune responses that several immune cells are involved such as macrophages, cytotoxic $\mathrm{T}$ cells, and natural killer cells $[4,45,46,50]$. Th2 cells are against extracellular multicellular parasites by releasing interleukin-4 (IL-4), IL-5, and IL-13 cytokines [51]. Furthermore, the interaction of dendritic cells with activated B cells may help dendritic cells to acquire unique abilities to promote polarization of Th2 [50]. The imbalance of Th1/Th2 may lead to immunological diseases, such as rheumatoid arthritis, type-1 diabetes, multiple sclerosis, and asthma [52]. Discovering the selective immunomodulators on different subsets of Th cells may be beneficial for the treatment of immune disorders.

In our presented data, most of the leaf extracts of Neolitsea species have low cytotoxicity. The leaf extracts of $N$. aciculata and $N$. villosa possessed differential immunomodulatory effects on Th1/Th2 balance. $N$. daibuensis leaf extracts slightly attenuated IL-4 production; in contrast, $N$. aciculata var. variabillima and N. hiiranensis leaf extracts attenuated both Th1 and Th2 cytokines. N. konishii leaf extracts dramatically suppressed IFN- $\gamma$ cytokine. The leaf extracts of N. aciculata var. variabillima and $N$. konishii differentially affect the functionality of different subsets of Th cells. Collectively, these Neolitsea species demonstrated selective immunomodulatory effects and the underlying mechanisms are needed for further study. Although there are a variety of immunosuppressive drugs such as cyclosporine $\mathrm{A}$, tacrolimus, daclizumab, and basiliximab to prevent the rejection of transplanted organs and tissues, 
autoimmune diseases, and inflammatory disorders [53], these drugs could cause systemic immunosuppression which greatly increases the risks of tumor formation and infections [54]. Hence, development of new therapeutic and preventive immunomodulators from medicinal plants to manage immune disorders is of great importance. There are many studies devoted to discover new immunomodulatory therapeutic compounds from natural plants with low toxicity [55-57]. p-Coumaryl alcohol-gamma-O-methyl ether (CAME) isolated from Alpinia galanga was selectively and substantially suppressed in IFN- $\gamma$ production in Th cells [11]. Farrerol, a new type of 2,3-dihydro-flavonoid isolated from the leaves of Rhododendron dauricum L, markedly suppressed concanavalin A- induced lymphocyte proliferation, Th1 and Th2 cytokine production, and differentiation of $\mathrm{T}$ helper cell populations [46]. Physalin $\mathrm{H}$ isolated from Physalis angulata exhibited an immunosuppressive activity on T-cell activation and proliferation by modulation of Th1/ Th2 immune balance [58]. There are 14 compounds from leaves of $N$. hiiranensis that exhibited anti-inflammatory activity to suppress the generation of superoxide anion from neutrophils [34]. Furthermore, four major metabolites of the leaves of $N$. hiiranensis, including elemane type, caryophyllene type, aromadendrene type, eudesmane type, and germacrane dilactone type, are the main effective anti-inflammatory constituents [34]. Caryophyllene oxide, a sesquiterpene compound from the leaves of N. hiiranensis, inhibited the influx of neutrophil into the inflammatory site and the activation of NF- $\kappa$ B pathway [59]. We recently reported that $N$. hiiranensis-derived terpenoids, including hiiranlactone $\mathrm{D}$, trans-phytol, and $\beta$-caryophyllene oxide, attenuated antigen-specific T helper 1 immunity [27]. Here, the leaf extracts of Neolitsea plants showed potential immunomodulatory activities on T-cell functionality. To further study the potential, immunomodulatory compounds from these plants will help to discover new immunomodulators.

\section{Conclusions}

The study demonstrated that most of the crude extracts of Taiwanese Neolitsea species, especially leaf extracts, were not toxic to primary splenocytes, but they are capable of decreasing IFN- $\gamma$ production without affecting IL-2 production by T cells. The selective Th1 immunomodulatory effects of the Neolitsea extracts indicate that the phytochemicals in these extracts have potential to be further evaluated and developed as immunomodulatory agents.

\section{Conflicts of Interest}

The authors declare no conflicts of interest. The authors are responsible for the content of this manuscript.

\section{Acknowledgments}

This work was supported by Grants MOST104-2320-B037-032-MY2 from the Ministry of Science and Technology of Taiwan and KMU-M106004 and KMU-M104011 from the Kaohsiung Medical University Research Foundation
(Kaohsiung City, Taiwan). The authors thank the Center for Research Resources and Development of Kaohsiung Medical University for providing the service of the LSRII flow cytometer.

\section{References}

[1] F. Belardelli and M. Ferrantini, "Cytokines as a link between innate and adaptive antitumor immunity," Trends in Immunology, vol. 23, pp. 201-208, 2002.

[2] K. A. Smith, K. J. Gilbride, and M. F. Favata, "Lymphocyte activating factor promotes T-cell growth factor production by cloned murine lymphoma cells," Nature, vol. 287, pp. 853-855, 1980.

[3] A. Billiau and P. Matthys, "Interferon-gamma: a historical perspective," Cytokine \& Growth Factor Reviews, vol. 20, pp. 97-113, 2009.

[4] M. Haak-Frendscho, S. A. Marsters, S. M. Chamow, D. H. Peers, N. J. Simpson, and A. Ashkenazi, "Inhibition of interferon-gamma by an interferon-gamma receptor immunoadhesin," Immunology, vol. 79, pp. 594-599, 1993.

[5] T. R. Mosmann, H. Cherwinski, M. W. Bond, M. A. Giedlin, and R. L. Coffman, "Two types of murine helper T cell clone. I. Definition according to profiles of lymphokine activities and secreted proteins," Journal of Immunology, vol. 136, pp. 2348-2357, 1986.

[6] C.-C. Wang, H.-L. Lin, S.-P. Wey, and T.-R. Jan, “Areca-nut extract modulates antigen-specific immunity and augments inflammation in ovalbumin-sensitized mice," Immunopharmacology and Immunotoxicology, vol. 33, pp. 315-322, 2011.

[7] I. J. Fuss, M. Neurath, M. Boirivant et al., "Disparate CD4+ lamina propria (LP) lymphokine secretion profiles in inflammatory bowel disease. Crohn's disease LP cells manifest increased secretion of IFN-gamma, whereas ulcerative colitis LP cells manifest increased secretion of IL-5," Journal of Immunology, vol. 157, pp. 1261-1270, 1996.

[8] A. S. Dayal, M. A. Jensen, A. Lledo, and B. G. Arnason, "Interferon-gamma-secreting cells in multiple sclerosis patients treated with interferon beta-1b," Neurology, vol. 45, pp. 2173-2177, 1995.

[9] B. Thiers, "Rituximab in autoimmune bullous diseases: mixed responses and adverse effects," Yearbook of Dermatology and Dermatologic Surgery, vol. 2008, 2008.

[10] E. Miserocchi, S. Baltatzis, M. R. Roque, A. R. Ahmed, and C. S. Foster, "The effect of treatment and its related side effects in patients with severe ocular cicatricial pemphigoid," Ophthalmology, vol. 109, pp. 111-118, 2002.

[11] E. S. Yu, H. J. Min, K. Lee et al., “Anti-inflammatory activity of p-coumaryl alcohol-gamma-O-methyl ether is mediated through modulation of interferon-gamma production in Th cells," British Journal of Pharmacology, vol. 156, pp. 11071114, 2009.

[12] A. Ziaei, M. Ramezani, L. Wright, C. Paetz, B. Schneider, and Z. Amirghofran, "Identification of spathulenol in Salvia mirzayanii and the immunomodulatory effects," Phytotherapy Research, vol. 25, pp. 557-562, 2011.

[13] H. N. D. Bao, H. Ushio, and T. Ohshima, "Antioxidative activity and antidiscoloration efficacy of ergothioneine in mushroom (Flammulina velutipes) extract added to beef and fish meats," Journal of Agricultural and Food Chemistry, vol. 56, pp. 10032-10040, 2008. 
[14] C. C. Wang, T. Y. Liu, S. P. Wey, F. I. Wang, and T. R. Jan, "Areca nut extract suppresses T-cell activation and interferon-gamma production via the induction of oxidative stress," Food and Chemical Toxicology, vol. 45, pp. 14101418, 2007.

[15] H. K. Kwon, J. S. Hwang, C. G. Lee et al., "Cinnamon extract suppresses experimental colitis through modulation of antigen-presenting cells," World Journal of Gastroenterology, vol. 17, pp. 976-986, 2011.

[16] H. Kang, Y. J. Oh, H. Y. Choi et al., "Immunomodulatory effect of Schizonepeta tenuifolia water extract on mouse Th1/Th2 cytokine production in-vivo and in-vitro," The Journal of Pharmacy and Pharmacology, vol. 60, pp. 901-907, 2008.

[17] F. Y. Chen, Y. P. Ye, H. X. Sun, X. Y. Li, and H. Shi, "Stemucronatoside $\mathrm{L}$, a pregnane glycoside from the roots of Stephanotis mucronata, inhibits Th1/Th2 immune responses in vitro," Chemistry \& Biodiversity, vol. 6, pp. 916-923, 2009.

[18] C. M. Ku and J. Y. Lin, "Anti-inflammatory effects of 27 selected terpenoid compounds tested through modulating Th1/Th2 cytokine secretion profiles using murine primary splenocytes," Food Chemistry, vol. 141, pp. 1104-1113, 2013.

[19] J. C. Liao, "Lauraceae in flora of taiwan," in Editorial Committee of the Flora of Taiwan, vol. 2, pp. 484-496, Taipei, Taiwan, 1996.

[20] W. Y. Wang, P. Ma, L. J. Xu, Y. Peng, and P. G. Xiao, "ChemInform abstract: chemical constituents and biological activities of plants from the genus Neolitsea," ChemInform, vol. 45, 2014.

[21] Y. Cao, X. L. Gao, G. Z. Su, X. L. Yu, P. F. Tu, and X. Y. Chai, "The genus Neolitsea of Lauraceae: a phytochemical and biological progress," Chemistry \& biodiversity, vol. 12, pp. 14431465, 2015.

[22] Editorial Board of Zhong Hua Ben Cao, Zhong Hua Ben Cao (China Herbal)," British Journal of Dermatology, vol. 156pp. 352-356, Shanghai Scientific Technology Press, Shanghai, China, 2007.

[23] H. S. Chang and I. S. Chen, "Chemical constituents and bioactivity of Formosan lauraceous plants," Journal of Food and Drug Analysis, vol. 24, 2016.

[24] S. C. Joshi, A. R. Verma, and C. S. Mathela, "Antioxidant and antibacterial activities of the leaf essential oils of Himalayan Lauraceae species," Food and Chemical Toxicology, vol. 48, pp. 37-40, 2010.

[25] C. M. Teng, S. M. Yu, S. S. Lee, F. N. Ko, M. J. Su, and T. F. Huang, "Vasoconstricting effect in rat aorta caused by thaliporphine isolated from the plant Neolitsea konishii K," European Journal of Pharmacology, vol. 233, pp. 7-12, 1993.

[26] K. S. Chen and Y. C. Wu, "Sesquiterpenoids from Neolitsea parvigemma: isolation, oxidation products and antiplatelet actions," Tetrahedron, vol. 55, 1999.

[27] Y. H. Cheng, I. S. Chen, Y. C. Lin, C. W. Tung, H. S. Chang, and C. C. Wang, "Attenuation of antigen-specific T helper 1 immunity by Neolitsea hiiranensis and its derived terpenoids," PeerJ, vol. 4, 2016.

[28] A. F. Majdalawieh, R. Hmaidan, and R. I. Carr, "Nigella sativa modulates splenocyte proliferation, Th1/Th2 cytokine profile, macrophage function and NK anti-tumor activity," Journal of Ethnopharmacology, vol. 131, pp. 268-275, 2010.

[29] C. T. Wu, K. S. Huang, C. H. Yang et al., "Inhibitory effects of cultured Dendrobium tosaense on atopic dermatitis murine model," International Journal of Pharmaceutics, vol. 463, pp. 193-200, 2014.
[30] C. H. Liao and J. Y. Lin, "Purification, partial characterization and anti-inflammatory characteristics of lotus (Nelumbo nucifera Gaertn) plumule polysaccharides," Food Chemistry, vol. 135, pp. 1818-1827, 2012.

[31] W. C. Lin and J. Y. Lin, "Five bitter compounds display different anti-inflammatory effects through modulating cytokine secretion using mouse primary splenocytes in vitro," Journal of Agricultural and Food Chemistry, vol. 59, pp. 184-192, 2011.

[32] W. C. Lin and J. Y. Lin, "Berberine down-regulates the Th1/Th2 cytokine gene expression ratio in mouse primary splenocytes in the absence or presence of lipopolysaccharide in a preventive manner," International Immunopharmacology, vol. 11, pp. 1984-1990, 2011.

[33] J. M. Dwyer and C. Johnson, "The use of concanavalin A to study the immunoregulation of human T cells," Clinical and Experimental Immunology, vol. 46, pp. 237-249, 1981.

[34] B. J. Liou, H. S. Chang, G. J. Wang et al., "Secondary metabolites from the leaves of Neolitsea hiiranensis and the antiinflammatory activity of some of them," Phytochemistry, vol. 72, pp. 415-422, 2011.

[35] A. J. Lengi, R. A. Phillips, E. Karpuzoglu, and S. A. Ahmed, "Estrogen selectively regulates chemokines in murine splenocytes," Journal of Leukocyte Biology, vol. 81, no. 4, pp. 10651074, 2007.

[36] R. Dai, R. A. Phillips, Y. Zhang, D. Khan, O. Crasta, and S. A. Ahmed, "Suppression of LPS-induced interferon-gamma and nitric oxide in splenic lymphocytes by select estrogenregulated microRNAs: a novel mechanism of immune modulation," Blood, vol. 112, no. 12, pp. 4591-4597, 2008.

[37] C. Tan, L. Wei, B. P. Vistica, G. Shi, E. F. Wawrousek, and I. Gery, "Phenotypes of Th lineages generated by the commonly used activation with anti-CD3/CD28 antibodies differ from those generated by the physiological activation with the specific antigen," Cellular \& Molecular Immunology, vol. 11, no. 3, pp. 305-313, 2014.

[38] S. S. Kim, J. E. Kim, C.-G. Hyun, and N. H. Lee, "Neolitsea aciculata essential oil inhibits drug-resistant skin pathogen growth and Propionibacterium acnes-induced inflammatory effects of human monocyte leukemia," Natural Product Communications, vol. 6, pp. 1193-1198, 2011.

[39] K. S. Chen, P. W. Hsieh, T. L. Hwang, F. R. Chang, and Y. C. $\mathrm{Wu}$, "Anti-inflammatory furanogermacrane sesquiterpenes from Neolitsea parvigemma," Natural Product Research, vol. 19, pp. 283-286, 2005.

[40] S. L. Wong, H. S. Chang, G. J. Wang et al., "Secondary metabolites from the roots of Neolitsea daibuensis and their antiinflammatory activity," Journal of Natural Products, vol. 74, pp. 2489-2496, 2011.

[41] H. C. Ku, S. Y. Lee, S.- S. Lee, and M. J. Su, "Thaliporphine, an alkaloid from Neolitsea konishii, exerts antioxidant, antiinflammatory, and anti-apoptotic responses in guinea pig during cardiovascular collapse in inflammatory disease," Journal of Functional Foods, vol. 26, 2016.

[42] S. S. Kim, C. G. Hyun, Y. H. Choi, and N. H. Lee, “Tyrosinase inhibitory activities of the compounds isolated from Neolitsea aciculata (Blume) Koidz," Journal of Enzyme Inhibition and Medicinal Chemistry, vol. 28, pp. 685-689, 2013.

[43] F. R. Chang, T. J. Hsieh, T. L. Huang et al., "Cytotoxic constituents of the stem bark of Neolitsea acuminatissima," Journal of Natural Products, vol. 65, pp. 255-258, 2002. 
[44] L. Wen-Shyong and D. Chang-Yih, "Sesquiterpene lactones from Neolitsea villosa," Phytochemistry, vol. 32, 1993.

[45] N. Hill and N. Sarvetnick, "Cytokines: promoters and dampeners of autoimmunity," Current Opinion in Immunology, vol. 14, pp. 791-797, 2002.

[46] Y. Xiong, S. Zhang, J. Lu et al., "Investigation of effects of farrerol on suppression of murine $\mathrm{T}$ lymphocyte activation in vitro and in vivo," International Immunopharmacology, vol. 16, pp. 313-321, 2013.

[47] N. Xu, X. Li, and Y. Zhong, "Inflammatory cytokines: potential biomarkers of immunologic dysfunction in autism spectrum disorders," Mediators of Inflammation, vol. 2015, Article ID 531518, 10 pages, 2015.

[48] E. Vivier, E. Tomasello, M. Baratin, T. Walzer, and S. Ugolini, "Functions of natural killer cells," Nature Immunology, vol. 9, no. 5, pp. 503-510, 2008.

[49] C. Saha, M. Das, E. Stephen-Victor, A. Friboulet, J. Bayry, and S. V. Kaveri, "Differential effects of Viscum album preparations on the maturation and activation of human dendritic cells and $\mathrm{CD}_{4+}$ T cell responses," Molecules, vol. 21, no. 7, 2016.

[50] M. S. Maddur and J. Bayry, "B cells drive Th2 responses by instructing human dendritic cell maturation," OncoImmunology, vol. 4, no. 5, 2015.

[51] V. Lazarevic and L. H. Glimcher, "T-bet in disease," Nature Immunology, vol. 12, pp. 597-606, 2011.

[52] K. S. Goudy, B. R. Burkhardt, C. Wasserfall et al., "Systemic overexpression of IL-10 induces CD4+CD25+ cell populations in vivo and ameliorates type 1 diabetes in nonobese diabetic mice in a dose-dependent fashion," Journal of Immunology, vol. 171, pp. 2270-2278, 2003.

[53] A. Johnston and D. W. Holt, "Immunosuppressant drugs-the role of therapeutic drug monitoring," British Journal of Clinical Pharmacology, vol. 52, pp. 61-73, 2001.

[54] T. Vial and J. Descotes, "Immunosuppressive drugs and cancer," Toxicology, vol. 185, pp. 229-240, 2003.

[55] B. Patwardhan and A. D. B. Vaidya, "Natural products drug discovery: accelerating the clinical candidate development using reverse pharmacology approaches," Indian Journal of Experimental Biology, vol. 48, pp. 220-227, 2010.

[56] A. L. Harvey, "Natural products in drug discovery," Drug Discovery Today, pp. 894-901, 2008.

[57] J. A. Beutler, "Natural products as a foundation for drug discovery," Current Protocols in Pharmacology, vol. 46, pp. 9.11.1-9.11.21, 2009.

[58] Y. Yu, L. Sun, L. Ma, J. Li, L. Hu, and J. Liu, "Investigation of the immunosuppressive activity of physalin $\mathrm{H}$ on $\mathrm{T}$ lymphocytes," International Immunopharmacology, vol. 10, pp. 290297, 2010.

[59] R. Medeiros, G. F. Passos, C. E. Vitor et al., "Effect of two active compounds obtained from the essential oil of Cordia verbenacea on the acute inflammatory responses elicited by LPS in the rat paw," British Journal of Pharmacology, vol. 151, pp. 618627, 2007.

[60] S. M. Yu, "Thaliporphine selectively inhibits expression of the inducible, but not the constitutive, nitric oxide synthase," Biochemical Journal, vol. 303, Part 1, pp. 289-294, 1994.
[61] C. L. Ho, P. C. Liao, E. I. C. Wang, and Y. C. Su, "Composition and antifungal activities of the leaf essential oil of Neolitsea parvigemma from Taiwan," Natural Product Communications, vol. 6, pp. 1357-1360, 2011.

[62] M. H. Lee, C. B. Jiang, S. H. Juan, R. D. Lin, and W. C. Hou, "Antioxidant and heme oxygenase-1 (HO-1)-induced effects of selected Taiwanese plants," Fitoterapia, vol. 77, pp. 109$115,2006$. 


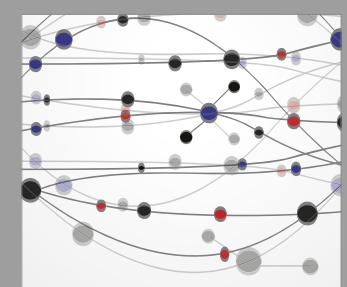

The Scientific World Journal
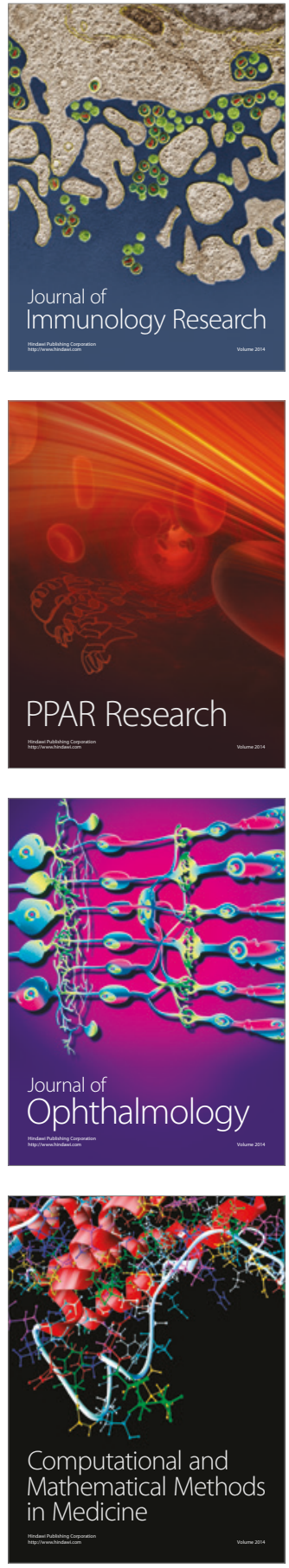

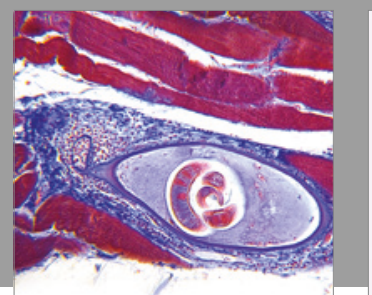

Gastroenterology Research and Practice
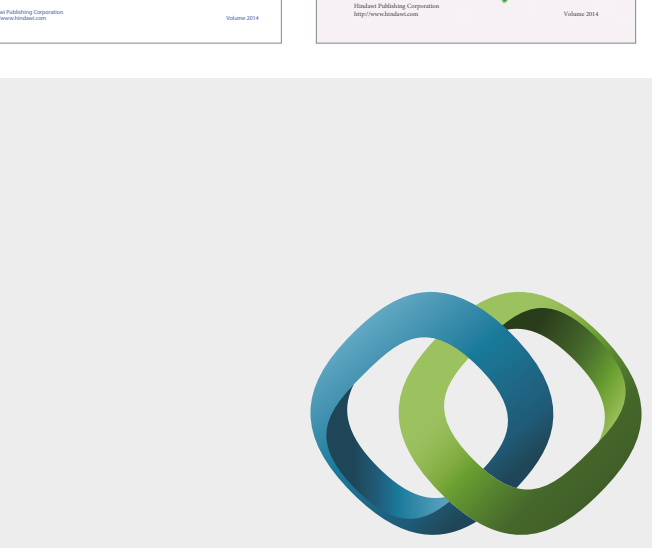

\section{Hindawi}

Submit your manuscripts at

https://www.hindawi.com
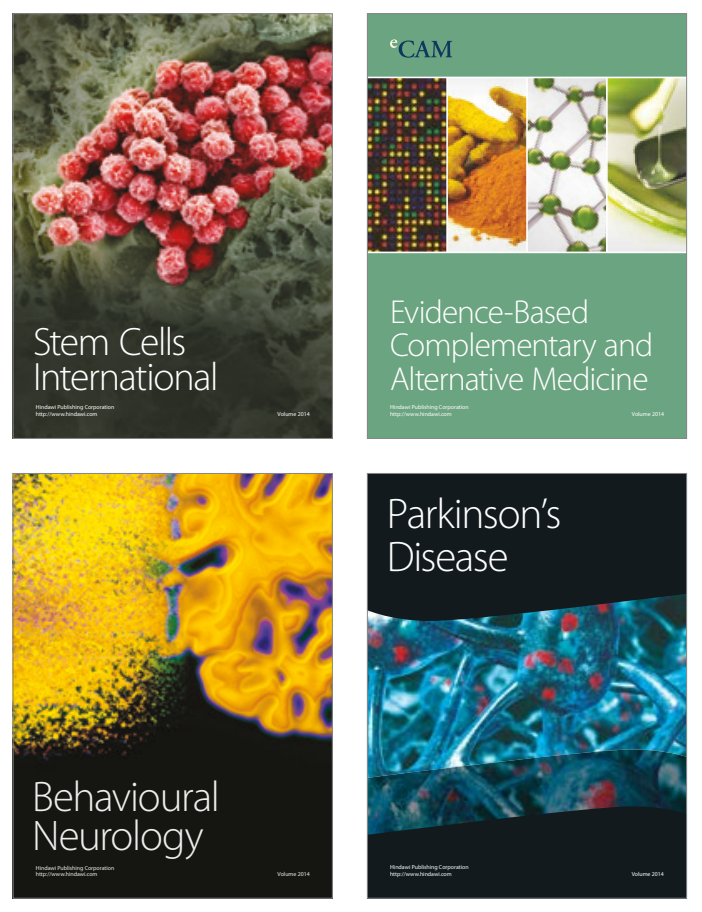
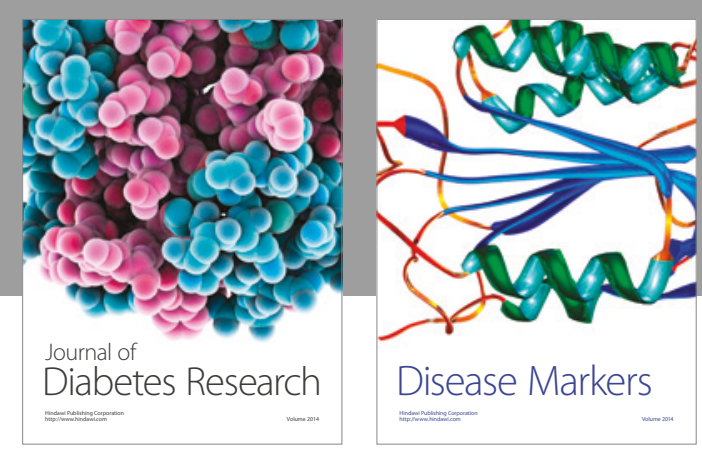

Disease Markers
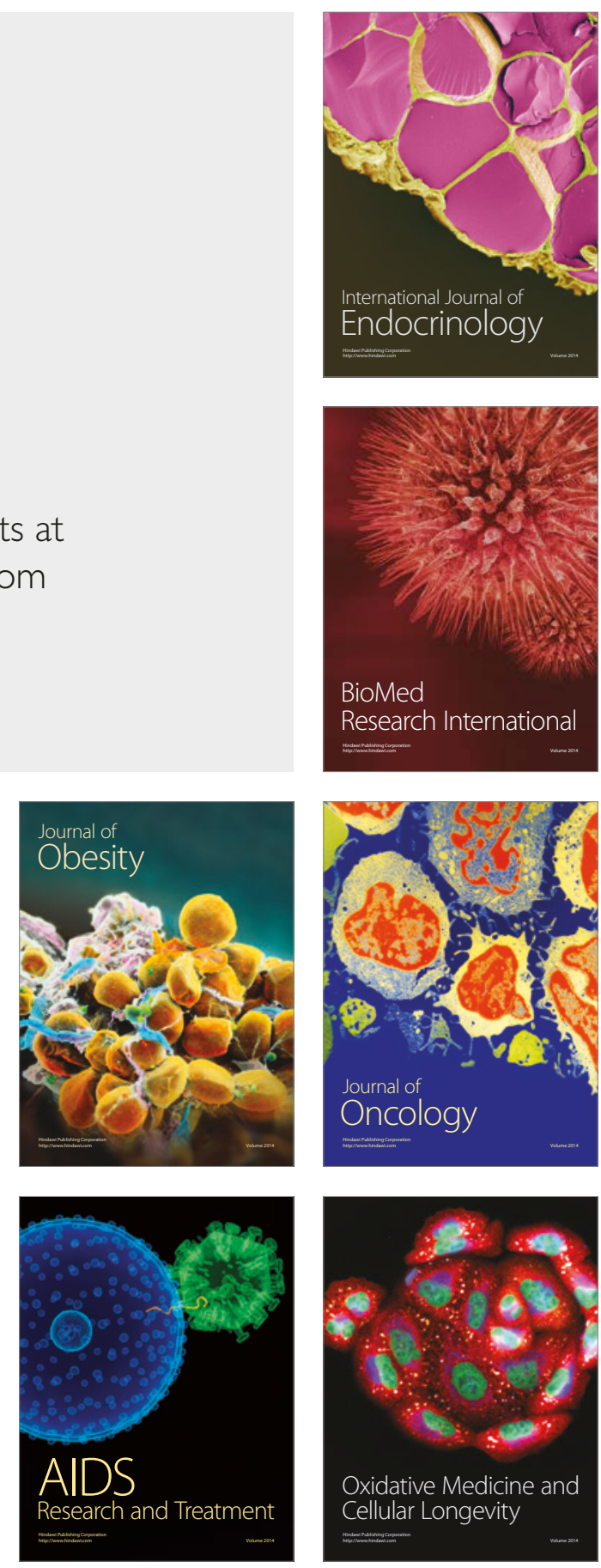